Recepción: 13 / 02 / 2018

Aceptación: 09 / 04 / 2018

Publicación: 05 / 06 / 2018
Ciencias de la Computación

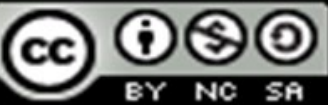

\title{
Influencia de la tecnología en el turismo: El internet
}

\author{
Influence of technology on tourism: The internet
}

Influência da tecnologia no turismo: a internet

\author{
Carlos R. Piguave-Mero ${ }^{\mathrm{I}}$
}

carlos.piguave@live.uleam.edu.ec

Correspondencia: carlos.piguave@live.uleam.edu.ec

\footnotetext{
${ }^{\text {I }}$ Magister en Gerencia Turística y Hotelera, Especialista en Diseño Curricular por Competencia, Analista de Sistemas, Docente de la Universidad Laica Eloy Alfaro de Manabí, Manta, Ecuador.
} 


\title{
Resumen
}

La presente investigación trata de la influencia de la tecnología en el sector del turismo con énfasis en el uso del internet. Asimismo, considera las proyecciones de la Organización Mundial del Trabajo con relación a la digitalización de los viajes, transporte y turismo en general, entre 2016 y 2025. Para su desarrollo se llevó a cabo una investigación de tipo documental bibliográfica. La búsqueda de información, paquetes, promociones, destinos, en general la planificación del viajero se realiza en su mayoría por medio del internet. Esta tendencia no sólo se limita a la etapa antes del viaje, también es mantenida durante y después del mismo, por tanto, es imperioso mantener al viajero conectado en tiempo real. Es innegable el avance del turismo a nivel mundial como actividad comercial y como pionero en la aplicación de las nuevas herramientas tecnológicas, lo que representa una ventaja para aquellos países que tienen la posibilidad de usarlas e invertir en este sector. Las proyecciones prometen un futuro halagador para el sector del futuro, todo depende de la inversión y el aprovechamiento de las herramientas tecnológicas emergentes como garantía de mantenerse vigente en la competencia y entre los primeros puestos del sector comercial a nivel mundial.

Palabras claves: influencia; tecnología; turismo; internet; viajero.

\begin{abstract}
This research deals with the influence of technology in the tourism sector with emphasis on the use of the internet. Likewise, it considers the projections of the World Labor Organization regarding the digitization of travel, transportation and tourism in general, between 2016 and 2025. For its development, a documentary bibliographic research was carried out. The search for information, packages, promotions, destinations, in general the planning of the traveler is done mostly through the internet. This trend is not only limited to the stage before the trip, it is also maintained during and after it, therefore, it is imperative to keep the traveler connected in real time. The advance of tourism worldwide as a commercial activity and as a pioneer in the application of new technological tools is undeniable, which represents an advantage for those countries that have the possibility of using them and investing in this sector. The projections promise a flattering future for the sector of the future, everything depends on the investment and
\end{abstract}


the use of the emerging technological tools as a guarantee of staying current in the competition and among the top positions of the commercial sector worldwide.

Keywords: Influence; Technology; Tourism; Internet; Traveler.

\section{Resumo}

Esta pesquisa trata da influência da tecnologia no setor de turismo com ênfase no uso da internet. Ele também considera as projeções da Organização Internacional do Trabalho sobre a digitalização de viagens, transporte e turismo em geral entre 2016 e 2025. Para o seu desenvolvimento foi realizado documentário pesquisa bibliográfica. A busca por informações, pacotes, promoções, destinos, em geral, o planejamento do viajante é feito principalmente através da internet. Esta tendência não se limita apenas ao estágio antes da viagem, ele também é mantida durante e após isso, portanto, é imperativo manter o viajante conectado em tempo real. Inegavelmente o avanço do turismo em todo o mundo como um negócio e como um pioneiro na aplicação de novas ferramentas tecnológicas, o que é uma vantagem para os países que têm a capacidade de usar e investir neste sector. Projeções prometem um futuro lisonjeiro para o futuro do setor, tudo depende de investimentos e alavancar ferramentas tecnológicas emergentes como garantia para ficar atualizado sobre a concorrência e entre os top setor comercial em todo o mundo.

Palavras chave: Influência; Tecnologia; Turismo; Internet; Viajante.

\section{Introducción}

En la actualidad el Turismo se ha posicionado como una de las primeras actividades del comercio internacional, siendo esta una de las principales fuerzas generadoras de ingresos y bienestar para muchos países.

En este orden de ideas, es importante mencionar que es una de las actividades comerciales que ha sido testigo y más ha sabido adoptar los diferentes cambios del desarrollo mundial a su favor, tales como la globalización, la tecnología, el internet, entre otros.

Esta versatilidad y flexibilidad ante los desafíos de la nueva era, le proporciona una característica propia del turismo en estos tiempos: la innovación, por medio de la cual se garantiza un constante 
crecimiento y avance a lo largo del tiempo, así mismo, conlleva a un mayor desarrollo de los países que invierten en dicha actividad.

Desde hace muchos años el turismo ha experimentado un crecimiento continuo y una onda diversificación, a tal punto de posicionarse entre uno de los sectores económicos que crece de manera más acelerada en el mundo. El turismo mundial y el desarrollo de los países guardan una estrecha relación, donde cada vez son más los nuevos destinos. Todo ello lo convierte en un motor clave del progreso socioeconómico. (Organización Mundial del Turismo, 2017)

Su posición de importancia varía de un país a otro, para algunos países su mejor fuente de ingresos es el petróleo, para otros la industria automotora, pero para muchos lo es el Turismo, incluso, independientemente del posicionamiento del nivel de ingreso económico que proporcione, la importancia radica en la suma de todas estas actividades para el beneficio económico de las regiones.

Se entiende como turismo a todas aquellas actividades que se relacionan con conocer o disfrutar de regiones o espacios en los que uno no vive de manera permanente. Su importancia radica en ser una actividad económica generadora de empleos, desarrollo de establecimientos gastronómicos y hoteleros, obras de infraestructura, crecimiento del transporte aéreo, terrestre o marítimo, entre otros. (Bembibre, 2011)

Es importante mencionar que el turismo permite resaltar las bondades culturales, históricas y naturales de una región y ofrecer al mundo esas características particulares para enriquecer las experiencias de los turistas o visitantes.

"En la era actual las empresas y las personas están conectadas, intercambian información y realizan transacciones. La combinación de plataformas digitales, las opiniones y contenidos creados por el usuario, la integración de las redes sociales, los sistemas de posicionamiento global y el uso de los macrodatos y la inteligencia artificial han transformado nuestra forma de gestionar, consumir y compartir la información”. (Organización Mundial del Turismo, 2018)

Todo ello consecuencia de los avances de la tecnología, en especial el internet, en este sentido, el presente trabajo de investigación plasma la importancia de estos cambios del desarrollo mundial y su influencia en el turismo, así como las proyecciones de este mercado. Es importante conocer 
y emplear las herramientas que nos brindan los avances de la nueva era a los fines de mejorar los servicios ofrecidos en la actividad del turismo y satisfacer mejor las necesidades del viajero, con el último propósito de mantenerse en la competencia y aumentar los ingresos de las regiones.

\section{Metodología}

Para llevar a cabo el presente trabajo se realizó una investigación de tipo documental bibliográfica, mediante la cual se plasmaron diversos aspectos relacionados con la influencia de los cambios del desarrollo mundial, tales como la globalización, la tecnología y el internet. Asimismo, las proyecciones de su implementación de estas herramientas en dicho mercado.

Tancara (1993), define la investigación documental como "una serie de métodos y técnicas de búsqueda, procesamiento y almacenamiento de la información contenida en los documentos, en primera instancia, y la presentación sistemática, coherente y suficientemente argumentada de nueva información en un documento científico, en segunda instancia" (p. 94)

\section{Resultados}

\section{La Tecnología en el Turismo}

La Universidad Internacional de Valencia (2018) define la tecnología como “...la ciencia que se aplica para dar solución a los problemas que se presentan en la sociedad, en diferentes sectores y se utiliza para desarrollar alternativas que ayudar a que las personas lleguen a tener una mejor vida...".

"El turismo fue uno de los primeros sectores en los que los procesos empresariales se digitalizaron a escala mundial, puesto que se puede considerar que las reservas de vuelos y hoteles en línea fueron unas de las iniciativas pioneras en el ámbito digital. A medida que la tecnología de la información y las comunicaciones (TIC) se iba convirtiendo en un fenómeno global, el turismo era uno de los primeros sectores que siempre iba adoptando las nuevas tecnologías y plataformas”. (Organización Mundial del Turismo, 2018)

"El uso de tecnologías como «el internet de las cosas», los servicios basados en la ubicación, la inteligencia artificial, la realidad virtual y aumentada y la tecnología de las cadenas de bloques, han generado una oferta turística más atractiva, eficiente, inclusiva y sostenible en términos 
económicos, sociales y medioambientales que su predecesora”. (Organización Mundial del Turismo, 2018)

Como resultado de la aplicación de las nuevas tecnologías en la actividad del turismo tenemos una transformación de dicha actividad que se traduce en la solución de problemas y la mejora de las ofertas.

A continuación, algunos de los aportes del uso de la tecnología en la actividad turística según la Organización Mundial del Turismo (2018):

\section{Facilitación de los viajes inteligentes.}

Un modelo integral de viajes inteligentes puede comprender visados, fronteras, procesos de seguridad e infraestructuras inteligentes, representarán una verdadera revolución en la industria del turismo. Con su traducción en términos tangibles, los pasajeros podrán reservar un vuelo y obtener la tarjeta de embarque en línea, llevarla en su teléfono móvil, pasar por controles de seguridad automatizados e incluso validar la tarjeta de embarque electrónicamente para subir al avión.

\section{Destinos Inteligentes.}

Suponen una estrategia en la que se observa la tecnología, la innovación, la sostenibilidad, la accesibilidad y la inclusión en todo el ciclo del viaje: antes, durante y después. Esta estrategia incluye a residentes y turistas, e integra en la planificación turística el multilingüismo, las distintas idiosincrasias culturales y la estacionalidad.

\section{Generación de Puestos de Trabajo.}

El futuro de los viajes está basado en la tecnología, por lo que los empleos del sector turístico requerirán aptitudes técnicas e interpersonales avanzadas para aplicar y gestionar de forma eficaz las iniciativas inteligentes.

Como en muchos sectores empresariales, en el turismo los empleos se ven cada vez más afectados por el uso de la tecnología, como consecuencia el reemplazo de personal en los procesos por automatización o robótica, lo que representa un reto para la empresa del turismo, la cual debe impulsar la educación y el entrenamiento en términos de desarrollo tecnológico, para 
asegurar la reinserción del personal en nuevos puestos de trabajo y la contratación de personal especializado, que impulse y promueva el uso de los avances tecnológicos.

\section{Repercusión del Internet en el Turismo}

(Brenes Leiva, 2012) cita a Castells (1999) quien considera que "la Internet está creando un mundo dividido entre los que tienen y los que no tienen Internet, este menciona que las diferencias de conectividad ocasionan que quienes no tengan acceso a Internet tengan una desventaja en el mercado de trabajo y que algunos territorios no conectados a Internet pierdan competitividad económica internacional y tiendan a ser bolsas crecientes de pobreza incapaces de sumarse al nuevo modelo de desarrollo". (p. 25)

De allí la necesidad imperiosa de adoptar estas nuevas tecnologías como parte de las estrategias organizacionales que permita a la empresa del turismo mantenerse competitiva en el mercado actual.

"El internet ha revolucionado la industria turística mucho más que cualquier otro factor en los últimos decenios. Asimismo, en vista de que cada vez más personas están conectadas entre sí, y tienen acceso a la abundante información que existe en línea, un número creciente de viajeros buscan información por Internet antes de tomar cualquier decisión con respecto a sus viajes". (Sakulsureeyadej, 2011)

Uno de los avances tecnológicos de la nueva era de mayor repercusión en muchas áreas es el internet, este se encuentra íntimamente relacionado con otras herramientas de la tecnología tales como las tecnologías de información y comunicación (TIC), especialmente "el turismo ha desarrollado una gran dependencia antes las TIC, pues antes de realizar un viaje, los turistas requieren información para planear y elegir entre múltiples opciones, y también observar la necesidad creciente de información durante y después del viaje”. (Acosta Carmona, 2015)

En la actualidad son muchos los viajeros que planifican sus viajes por medio del internet, estos procesos automáticos al alcance de todos han desplazado la actividad de planificación proporcionada por las agencias de viajes. La comodidad, fácil acceso y una amplia oferta de información son algunos de los aportes del internet en la elección y planificación de un destino. En la actualidad podemos acceder a través de un teléfono inteligente y realizar actividades que 
van desde búsquedas de información para la elección del destino, hasta compras de boletos, búsqueda de promociones y reservaciones de hoteles, todo ello desde la comodidad del hogar o bien sentados en un café.

Vidal (2018) cita un estudio de Google Travel cuyos resultados concluyen que "el $74 \%$ de los viajeros de placer planean sus viajes por Internet, mientras que solo el 13\% lo hace ya a través de agencias de viaje".

Por otra parte, no podemos mencionar los avances que ha proporcionado el internet a la industria del turismo sin mencionar el internet de las cosas, todos y cada uno de los objetos que funcionan con internet, la actividad hotelera, los vehículos de alquiler, son algunas de las actividades que más los usan, desde localizadores hasta aplicaciones que permiten al visitante controlar muchas cosas y al servicio turístico ofrecer mayores comodidades.

"Los nuevos desarrollos tecnológicos ligados al Internet de las Cosas, al Big Data/Open Data, a la Inteligencia Artificial, Blockchain o la Realidad Virtual abren la puerta a nuevos procesos, nuevas formas de hacer las cosas o nuevas estrategias para captar y fidelizar al cliente como nunca antes pudimos imaginar. El mundo del marketing, ventas y programas de fidelización entran en una nueva era del conocimiento, de la analítica y del aprendizaje continuo". (López de Ávila, 2017)

Otra ventaja que valoran los viajeros en la actualidad en la oferta de servicios hoteleros es el wifi, asegurarse una buena conexión a internet con el propósito de mantenerse informado y con el uso de toda la tecnología disponible en su teléfono inteligente es un factor determinante de esta nueva era para el turista, además de asegurarse compartir las experiencias de su viaje a través de fotos y comentarios.

\section{Proyecciones}

La digitalización en la aviación, los viajes y el turismo (Entre 2016 y 2025), según la Organización Mundial del Turismo (2018), se estima:

- Genere ingresos de hasta 305.000 millones de dólares de los EE.UU. en el sector turístico debido al aumento de la rentabilidad. 
- Transfiera ingresos del orden de 100.000 millones de dólares de los EE.UU. de ámbitos tradicionales del sector a nuevos competidores.

- Aporte beneficios valorados en 700.000 dólares de los EE.UU. en favor de los consumidores y la sociedad en general, mediante la reducción de la huella ambiental, las mejoras en materia de seguridad y protección y el ahorro de tiempo y costos.

- Provoque un claro reemplazo de los empleos actuales, en parte compensado por la creación de empleos cualificados de nueva generación dentro y fuera del sector turístico y otros sectores afines.

\section{Conclusiones}

El turismo en la actualidad ha sufrido una serie de transformaciones las cuales han permitido adaptarlo a las exigencias de esta era moderna. El desarrollo de la humanidad trae consigo una serie de fenómenos que obligan a las empresas del sector económico a tomar una estructura flexible que les permita cumplir con las demandas del día a día. Los avances tecnológicos representan una gran parte de la influencia de los cambios para el futuro que ha creado una nueva industria del turismo a nivel mundial.

Siendo el turismo una de las principales fuentes de ingresos de muchos países a nivel mundial y siendo pilar fundamental de su desarrollo cultural, histórico y de los recursos naturales de cada región, además de representar a una de las actividades económicas más sustentables y sostenibles, es imperioso prestar atención a los mencionados cambios que han venido transformando no sólo la actividad del turismo sino el comportamiento de la humanidad.

El propósito fundamental de cualquier empresa está orientado a generar utilidad, para nadie es un secreto que en la era actual existe una competencia sin precedentes, lo que lleva a que sólo aquellos que saquen provecho de las herramientas tecnológicas, la globalización, el internet y hasta lo que en determinado momento sea una dificultad o problema y lo transforme en beneficio de la organización, mejores productos, mejores ofertas, mejores servicios, mayor atención, entre otros, serán factores determinantes para mantenerse entre los líderes de los mercados.

El turismo no escapa a esta realidad, en un mundo donde el comportamiento humano y sus actividades, preferencias, gustos, cambian constantemente de la mano de las nuevas tecnologías, 
es imperioso que esta industria vaya a la par con estos cambios y sea no sólo testigo sino partícipe de este desarrollo.

Son cada vez más los usuarios, viajeros o turistas que planifican su experiencia de viaje por medio del uso del internet, búsqueda de destinos, información, paquetes, promociones, temporadas, compra de boletos, reservaciones de hoteles, de vehículos, paquetes de excursión, entre otros.

Así mismo, el internet de las cosas juega un papel fundamental que debe considerarse en el servicio de turismo, el viajero quiere estar conectado a la red en cuanto más le sea posible, y las empresas deben ofrecer un mejor servicio proporcionándoles esa opción en muchos de los objetos físicos que se encontrarán a lo largo de su viaje.

La conexión wifi también tiene un papel preponderante ya que colabora con la interacción en tiempo real que busca el viajero ya sea con empresas hoteleras, de vehículos, con amigos y familiares. El viajero desea compartir su experiencia con el mundo por medio de la valoración de los servicios usados y la publicación de fotos y comentarios de su viaje, a través de la economía y la buena conexión que le pueda brindar un servicio wifi.

En cuanto a las proyecciones realizadas por la Organización Mundial del Turismo (OMT) ante la digitalización de la industria de turismo, las estimaciones son favorables y muy a pesar de que se realizaron sólo para los Estados Unidos, es innegable que el desarrollo del turismo como empresa generadora de ingresos para muchos países va en ascenso y se prevé continúe creciendo en la medida en que se adapte a las exigencias actuales y saque el mayor provecho de los avances de la tecnología.

Lo que hace pensar cuál será el destino de aquellos países menos desarrollados que no pueden acceder a estas nuevas tecnologías, para ellos estará reservado un menor posicionamiento dentro del mercado del turismo, al igual que para aquellos países que no inviertan en este sector.

\section{Referencias Bibliográficas}

Acosta Carmona, A. (01 de junio de 2015). EOI. Recuperado el 08 de Agosto de 2018, de http://www.eoi.es/blogs/embatur/2015/06/01/las-tics-y-su-importancia-para-el-turismo/ 
Bembibre, C. (29 de mayo de 2011). Importancia.org. Recuperado el 08 de Agosto de 2018, de https://www.importancia.org/turismo.php

Brenes Leiva, R. (2012). El Turismo en la Globalización. Círculo de Cartago, 4, 30. Recuperado el 09 de Agosto de 2018, de http://www.circulodecartago.org/wp-content/uploads/2012/08/ElTurismo-en-la-Globalizaci\%C3\%B3n.pdf

López de Ávila, A. (18 de octubre de 2017). Turismo e innovación: el nuevo modelo turístico del siglo XXI. América Economía. Recuperado el 09 de Agosto de 2018, de https://www.americaeconomia.com/analisis-opinion/turismo-e-innovacion-el-nuevo-modeloturistico-del-siglo-xxi

Organización Mundial del Turismo. (2017). UNWTO Organización Mundial del Turismo. Recuperado el 08 de Agosto de 2018, de http://www2.unwto.org/es/content/por-que-el-turismo Organización Mundial del Turismo. (2018). UNWTO Organización Mundial del Turismo. Recuperado el 08 de Agosto de 2018, de http://wtd.unwto.org/es/content/dmt-2018-tecnologiaen-el-turismo

Organización Mundial del Turismo. (2018). UNWTO Organización Mundial del Turismo. Recuperado el 08 de Agosto de 2018, de http://wtd.unwto.org/es/content/dmt-2018-turismo-enla-era-digital

Organización Mundial del Turismo. (2018). UNWTO Organización Mundial del Turismo. Recuperado el 08 de Agosto de 2018, de http://wtd.unwto.org/es/content/dmt-2018-la-cuartarevolucion-industrial

Sakulsureeyadej, A. (2011). UNWTO Organización Mundial del Turismo. Recuperado el 08 de Agosto de 2018, de http://cf.cdn.unwto.org/sites/all/files/pdf/omt_amreports_numero1_tecnologiaturismo_esp.pdf

Tancara, C. (1993). La Investigación Documental. Temas Sociales (17), 91-106. Obtenido de http://www.revistasbolivianas.org.bo/pdf/rts/n17/n17a08.pdf 
Universidad Internacional de Valencia. (21 de marzo de 2018). VIU Universidad Internacional de Valencia. Recuperado el 08 de Agosto de 2018, de https://www.universidadviu.com/que-es-latecnologia/

Vidal, B. (01 de agosto de 2018). We Are Marketing. Recuperado el 08 de Agosto de 2018, de https://www.wearemarketing.com/es/blog/turismo-y-tecnologia-como-la-tecnologia-revolucionael-sector-turistico.html 